Ann. Biol. anim. Bioch. Biophys., I976, 16 (I), 37-42.

\title{
DÉMONSTRATION PAR LE ROUGE DE RUTHÉNIUM D'INVAGINATIONS PROFONDES DE LA MEMBRANE PLASMIQUE APICALE DES ENTÉROCYTES DANS L'INTESTIN POSTÉRIEUR CHEZ LA TRUITE ARC-EN-CIEL
}

\author{
P. BERGOT \\ avec la collaboration technique de Nelly KaUshiK \\ Laboratoire de Nutrition des Poissons, \\ Centre national de Recherches zootechniques, I. N. R. A., \\ 78350 Jouy en Josas \\ RÉSUMÉ,
}

\begin{abstract}
Chez des truites arc-en-ciel (Salmo gairdnevii RICH.) de $15 \mathrm{~cm}$ de longueur, l'utilisation du rouge de ruthénium a permis de visualiser des invaginations tubulaires profondes de la surface cellulaire libre à travers la zone apicale des entérocy tes de l'intestin postérieur. Ces invaginations sont interprétées comme le signe d'une activité de pinocytose qui persiste, au-delà du stade alevin, chez la Truite en croissance.
\end{abstract}

\section{INTRODUCTION}

D'après yamamoto (Ig66), les cellules épithéliales absorbantes de l'intestin de Truite arc-en-ciel seraient dépourvues des invaginations et des vésicules de pinocytose qui caractériseraient les entérocytes de l'intestin postérieur du Poisson rouge (Carassius auratus L.). Pour cet auteur, les mécanismes d'absorption seraient différents chez le Poisson rouge, poisson sans estomac, et chez la Truite qui a un estomac.

Des observations ultérieures ont confirmé la présence d'images de pinocytose, au niveau de l'intestin postérieur, chez le Poisson rouge, aussi bien au stade de l'alevin de $6 \mathrm{~mm}$ de long (IwaI, I968 a) que chez des individus plus âgés (GaUTHIER et LANDIS, I972), et ont montré également leur existence chez l'alevin de Truite de 20-22 $\mathrm{mm}$ de long, avant la résorption de la vésicule vitelline (IWAI, I968 b). Ces 
auteurs ont suggéré que la pinocytose permettait l'absorption des protéines alimentaires suivie de leur dégradation intracellulaire et pouvait remplacer la digestion gastrique, en milieu acide, toujours absente chez le Poisson rouge et faible chez l'alevin de Truite arc-en-ciel.

Yамамото (I966) n'ayant pas fait de distinction entre intestin antérieur et intestin postérieur, nous avons recherché si les différences ultrastructurales décrites entre les entérocytes de ces deux niveaux chez 1'alevin par IwAI (Ig68 b) se retrouvaient chez la Truite plus âgée. Celle-ci possède un intestin pourvu de cæca pyloriques dans sa partie antérieure et de valvules internes, circulaires ou spirales, dans sa partie postérieure.

Pour démontrer avec certitude la continuité des invaginations de la membrane plasmique apicale avec la lumière intestinale, nous avons utilisé le rouge de ruthénium (LUFT, I97I).

En présence de tétroxyde d'osmium, le rouge de ruthénium contraste les mucopolysaccharides, associés à la face externe de la membrane plasmique, avec lesquels il vient en contact et peut ainsi servir de marqueur de la surface cellulaire libre (CHAMBERS, I973). Il a permis, en particulier, de contraster les invaginations de pinocytose dans les entérocytes du Poisson rouge (Yamamoto, 1972).

\section{MATÉRIEI, ET MÉTHODES}

Des truites arc-en-ciel (Salmo gairdnerii $\mathrm{RICH}$ ) de $15 \mathrm{~cm}$ de longueur, élevées à $\mathrm{I}^{\circ} \mathrm{C}$, sont sacrifiées $24 \mathrm{~h}$ après leur dernier repas. Après introduction du fixateur (glutaraldéhyde 3 p. I dans du tampon cacodylate, $\mathrm{pH} 7,3$ ) dans la lumière intestinale, des segments de l'intestin antérieur (au niveau de l'implantation des derniers cæca pyloriques) et de l'intestin postérieur sont isolés par des ligatures et plongés dans le même fixateur. Après I heure des échantillons de muqueuse sont postfixés par le tétroxyde d'osmium à $2 \mathrm{p}$. Ioo dans le même tampon. Du rouge de ruthénium est ajouté aux deux liquides fixateurs ou seulement au second (concentration finale 0,05 p. IOo).

Les coupes ultrafines des tissus inclus dans l'Épon sont observées au microscope électronique après coloration éventuelle par l'acétate d'uranyle et le citrate de plomb.

\section{RÉSULTATS}

Après traitement par le rouge de ruthénium, ajouté aux deux liquides fixateurs ou seulement au fixateur osmique, on observe sur la surface apicale des entérocytes un dépôt dense aux électrons, contrastant nettement avec le reste du tissu même si celui-ci est coloré par l'uranyle et le plomb. Ce dépôt recouvre la surface externe des microvillosités qui présentent une structure et une disposition régulière classiques (fig. I, 2, 3 et 4). Les trois feuillets de la membrane plasmique restent visibles (fig. 5). On n'observe pas de dépôt sur les membranes plasmiques latérales des entérocytes au-dessous des jonctions étroites, sauf en cas de rupture des liaisons intercellulaires. De même, les organites cellulaires ne sont pas contrastés sauf en cas de lésion de la membrane plasmique. 
Des différences frappantes entre les entérocytes de l'intestin antérieur et ceux de 1'intestin postérieur apparaissent dans la zone apicale :

- Dans les entérocytes de l'intestin antérieur, la zone apicale forme une couche homogène, pauvre en structures membranaires (fig. 2 et 4 ).

- Au contraire, dans les entérocytes de l'intestin postérieur, on observe, à la base des microvillosités, des invaginations profondes de la membrane plasmique formant des puits tubulaires qui s'enfoncent perpendiculairement à la surface cellulaire dans la zone apicale. La profondeur de ces puits est du même ordre de grandeur que la hauteur des microvillosités. Leur contraste superficiel, dû au rouge de ruthénium permet d'identifier ces invaginations quelle que soit l'orientation de la coupe (fig. I).

La zone sous apicale contient, outre les organites habituels, de nombreuses vésicules accompagnées de microtubules et de faisceaux de micro filaments généralement parallèles à l'axe des microvillosités. Dans la région supranucléaire, on observe des vésicules de grande taille (diamètre supérieur à $\mathrm{I} \mu$ ) dont le contenu est peu dense aux électrons à côté de vésicules à contenu plus dense qui ont l'aspect de lysosomes après coloration à l'uranyle et au plomb.

\section{DISCUSSION}

A l'encontre de Yамамото (I966), nos observations montrent la présence dans les entérocytes de Truite d'invaginations et de vésicules tout à fait comparables à celles décrites chez le Poisson rouge et chez 1'Alevin de truite (IWAI, I968 $b$ ). La présence de ces invaginations ne paraît donc pas liée à l'absence ni à la faiblesse de la digestion gastrique, contrairement aux hypothèses précédentes, mais à une différenciation régionale de l'intestin. Les observations de Yamamoto (Ig66) sur la Truite s'appliquent, en effet, aux entérocytes de l'intestin antérieur, où les invaginations sont rares et non à ceux de l'intestin postérieur, où elles sont nombreuses.

Les particularités ultrastructurales de 1'intestin postérieur suggèrent que celui-ci ne joue pas, dans l'absorption, le même rôle que 1'intestin antérieur. On a ainsi signalé, chez la Truite, des différences entre ces deux parties de l'intestin dans le transport, in vitro, de l'eau, du sodium (BENSHALA-TALET et al., r974) et du calcium (Chartier, I974).

Dans 1'intestin antérieur, la pinocytose paraît peu importante. Ceci est en accord avec les observations morphologiques antérieures montrant que l'absorption des lipides, qui a lieu à ce niveau, ne se fait pas par pinocytose chez la Truite (IwaI, I968 $b$; BERGo't et FleCHON, I970), le Poisson rouge (IWAI, I968 $a$; GaUthIER et LANDis, I972) et la Carpe (NOAILLAC-DEPEYRE et GAS, I974).

Au niveau de l'intestin postérieur, on a montré, chez le Poisson rouge, une possibilité d'absorption de macromolécules protéiques (peroxydase exogène) par pinocytose (GAUTHIER et LANDIS, I972). Une situation analogue existe au niveau de l'iléon chez le Mammifère nouveau-né, correspondant à l'absorption non spécifique et à la dégradation des protéines (GRaney, I968; Rodewal,D, I973). Dans 1'intestin postérieur de Truite, la nature du matériel transporté et son devenir (absorption ou sécrétion) ne 
sont pas connus, mais il est probable que les invaginations correspondent également à une activité de pinocytose. Dans cette hypothèse, la possibilité d'absorber des macromolécules protéiques par pinocytose, qui est souvent considérée comme temporaire chez le Mammifère après la naissance, serait un phénomène durable pour la partie postérieure de l'intestin de truite et de cyprinidé (NOAILIAC-DEPEYRE et GAS, I973).

Reçu pour publication en juillet 1975.

\title{
SUMMARY
}

\author{
DEMONSTRATION WITH RUTHENIUM RED OF DEEP \\ INVAGINATIONS IN THE APICAL PLASMA MEMBRANE \\ OF ENTEROCYTES IN RAINBOW TROUT POST-INTESTINE
}

After staining with Ruthenium red, deep tubular invaginations of the cell surface were observed through the terminal webs of enterocytes in the distal part of the intestine in $15 \mathrm{~cm}$ long Rainbow trouts. These invaginations were interpreted as an evidence of persistence of pinocytosis beyond the larval stage in the growing Trout.

\section{RÉFÉRENCES BIBLIOGRAPHIQUES}

Bensahla-Talet A., Porte-Nibelle J., Lahlou B., I974. Le transport de l'eau et du sodium par l'intestin isolé de la Truite Salmo iridetus au cours de l'adaptation à l'eau de mer, C. R. Acad. Sc., Paris, 278, D, 254I-2544.

Bergot P., Flechon J. E., ig7o. Forme et voie d'absorption intestinale des acides gras à chaîne longue chez la Truite arc-en-ciel (Salmo gairdnerii RICH.) Ann. Biol. anim. Bioch. Biophys., 10, 459-472.

Chambers V. C., I973. The use of ruthenium red in an electron microscope study of cytophagocytosis. J. Cell. Biol., 57, $874-878$.

Chartier M. M., I974. Osmorégulation et proténe liant le calcium (Ca BP) des nuqueuses intestinales et branchiales de Truite arc-en-ciel, Salmo gairdnerii. C. R. Acad. Sc., Paris, 279, D, 927-930.

Gauthier Gi F., Landis S. C., 1972. The relationship of ultrastructural and cytochemical features to absorptive activity in the goldfish intestine. Anat. Rec., 172, 675-702.

Graney D. O., rg68. The uptake of ferritin by ileal absorptive cells in suckling rats. An electron microscope study. Am.J. Anat., 123, 227-254.

IwaI T., I968 a. The comparative study of the digestive tact of teleost larvae. V. Fat absorption in the gut epithelium of goldfish larvae. Bull. Jap. Soc. Sci. Fish., 34, 973-978.

Iwar T., I968 b. Fine structure and absorption patterns of intestinal epithelial cells in rainbow trout alevins. Z. Zellforsch. Mikrosk. Anat., 72, 366-379.

LuFT J. H., I97ı. Ruthenium red and violet. II. Fine structural localization in animal tissues. Anat. Rec., 171, 369-416.

Noaillac-Depeyre J., Gas N., 1973. Absorption of protein macromolecules by the enterocytes of the carp (Cyprinus carpio L.). Z. Zellforsch. Mikrosk. Anat., 146, 525-541.

Noaillac-Depeyre J., Gas N., T974. Fat absorption by the enterocytes of the carp (Cyprinus carpio L. Cell Tissue Res., 155, 353-365.

RODEWALD R., 1973. Intestinal transport of antibodies in the newborn rat. J. Cell Biol., 58, I89-2II.

Yамамото T., r966. An electron microscope study of the columnar epithelial cell in the intestine of fresh water teleosts : Goldfish (Carassius auratus) and rainbow trout (Salmo irideus). Z. Zellforsch. Mikrosk. Anat, 72, 66-87.

Yамамото T. 1972. Absorption across the plasma membrane of the intestinal absorptive cells. Acta Histochem. Cytochem., 5, 266-268. 


\section{PLANCHES}




\section{PLANCHE I}

\section{Zone apicale des entérocytes de Truite}

(double fixation avec rouge de ruthénium, double coloration)

FiG. I. - Intestin postérieur

(coupe parallèle à l'axe des microvillosités) ( $\times 28$ ooo)

Le dépôt de rouge de ruthénium, très dense aux électrons, recouvre la surface des microvillosités $(M)$ et celle des invaginations (I) de la membrane plasmique libre. Quand elles sont coupées obliquement, ces invaginations ont l'aspect de vésicules isolées. Leur contraste superficiel montre cependant qu'elles communiquent avec la lumière intestinale $(L)$. La zone apicale $(Z)$ contient également des vésicules (v) non contrastées, des microfilaments (F) et des microtubules (T).

FIG. 2. - Intestin antérieur

(coupe parallèle à l'axe des microvillosités) ( $\times 25000)$

I a zone apicale $(Z)$ est dépourvue d'invaginations et de vésicules. Le rouge de ruthénium contraste fortement la surface cellulaire libre sur les microvillosités $(M)$. Il ne pénètre pas dans les espaces intercellulaires. 

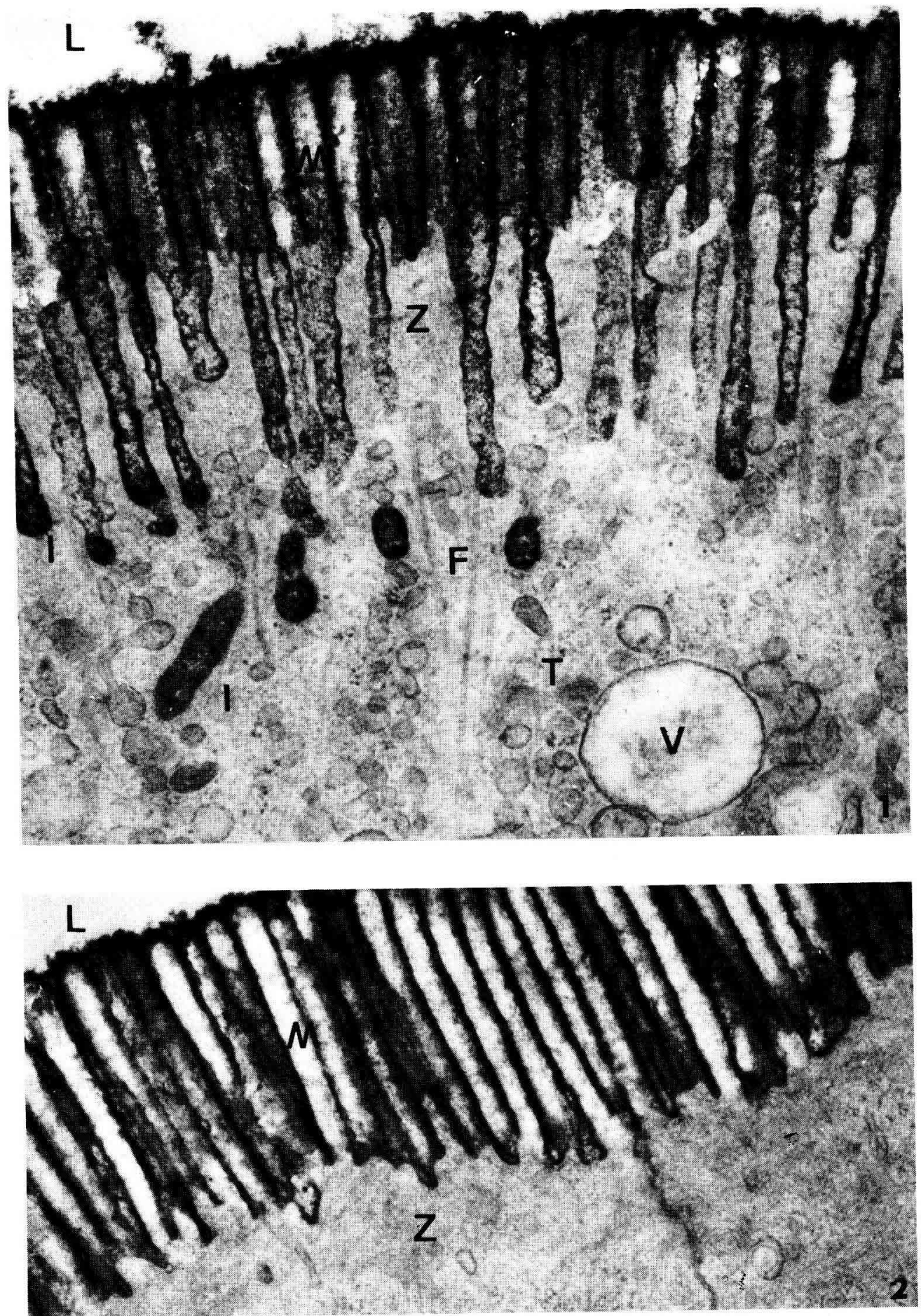


\section{PLANCHE II}

Microvillosités (M) et zone apicale $(Z)$ des entérocytes de Truite (double fixation avec rouge de ruthénium et double coloration).

FIG. 3

Intestin postérieur (coupe oblique presque perpendiculaire à l'axe des microvillosités). $\times 17000$.

De nombreuses invaginations (I) donnent un aspect irrégulier à la surface cellulaire où sont implantées les microvillosités.

FIG. 4.

Intestin antérieur (même orientation de coupe que la fig. 3). $\times 18000$.

Les invaginations sont rares dans la zone apicale $(Z)$ qui est bien délimitée.

Fig. 5.

Intestin antérieur (coupe perpendiculaire à l'axe des microvillosités). $\times 200000$.

Le rouge de ruthénium colore les mucopolysaccharides (glycocalyx) associés au feuillet externe de la membrane plasmique (P) dont la structure trilaminaire est visible. Il ne colore pas l'intérieur des microvillosités $(M)$. 

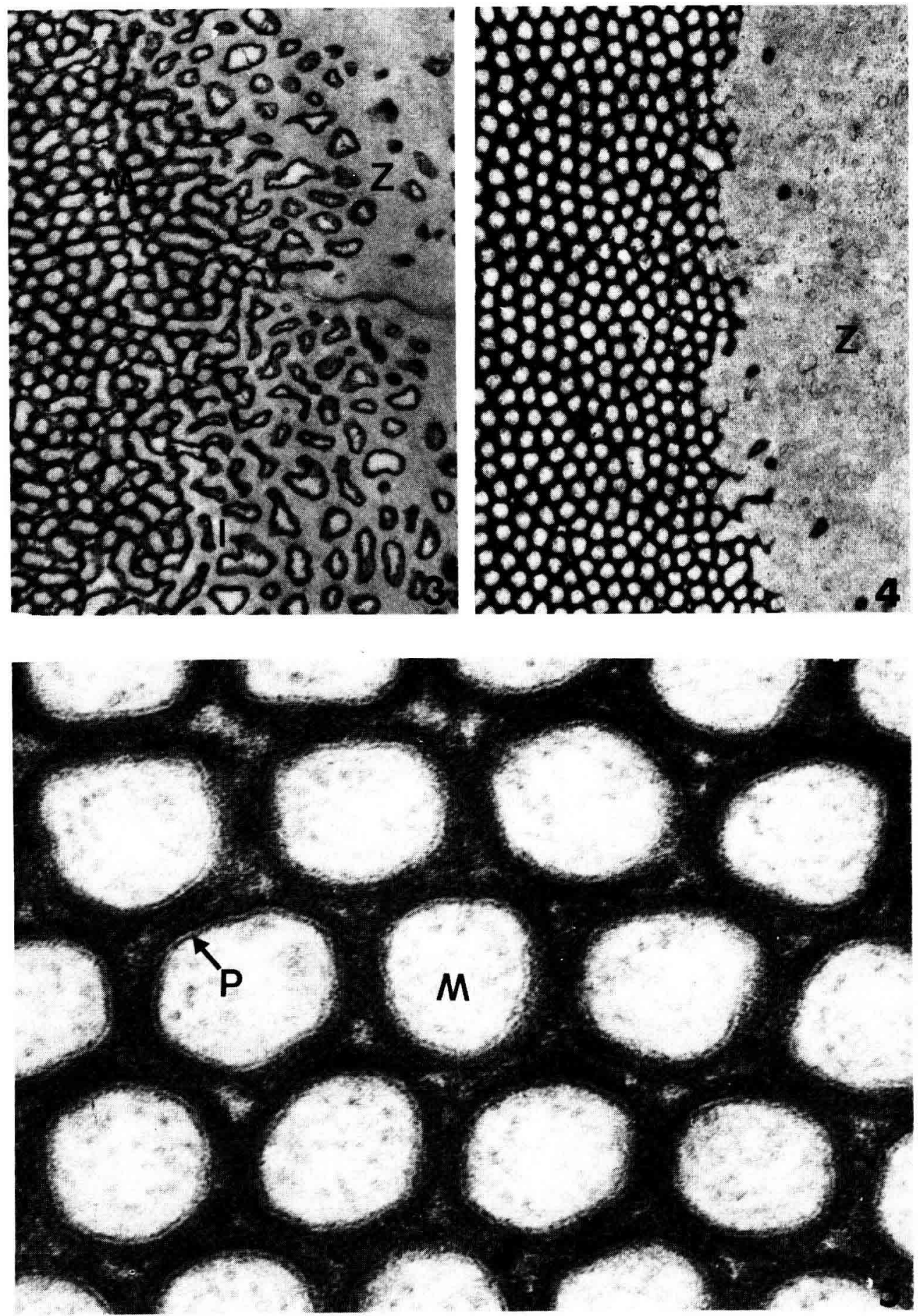